\title{
COMPORTAMENTO SAZONAL DOS PREÇOS DO FARELO DE SOJA NO ESTADO DE SÃO PAULO E OS PREÇOS EM ILHA SOLTEIRA (SP)
}

\author{
João Alberto Fischer Filho ${ }^{1}$, Elaine Mendonça Bernardes ${ }^{2}$, Nadia Maria Poloni $^{3}$
}

\footnotetext{
${ }^{1}$ Mestrando em Agronomia - Ciência do Solo, Faculdade de Ciências Agrárias e Veterinárias / UNESP Campus de Jaboticabal, email: joaofischer16@gmail.com

${ }^{2}$ Professor Assistente Doutor, UNESP Campus Experimental de Dracena, email: emb@dracena.unesp.br

${ }^{3}$ Mestrando em Agronomia - Sistemas de Produção, Faculdade de Engenharia / UNESP Campus de Ilha Solteira, email: nadia_poloni@ hotmail.com
}

RESUMO: A questão da sazonalidade é um aspecto que assume grande relevância, afetando diretamente a quantidade ofertada de produtos no mercado e, consequentemente, seus preços. Neste sentido, os produtores rurais têm de analisar os preços dos fatores de produção visando reduzir seus custos. Particularmente para a produção de carnes, os preços do farelo de soja são relevantes. Desta maneira, objetivou-se neste estudo: i) analisar o comportamento de preços do farelo de soja no município de Ilha Solteira (SP); ii) comparálos a médias do Estado de São Paulo; e iii) calcular o índice de sazonal para o estado, pelo Método da Média Geométrica Móvel Centralizada, descrito em Hoffman (1980). Conclui-se que: i) os preços em Ilha Solteira foram sempre superiores (exceto em julho e agosto de 2012) que os preços do farelo de soja pagos pelos produtores do Estado de São Paulo; ii) os preços médios pagos pelo farelo de soja por produtores paulistas apresentaram comportamento sazonal, iii) o comportamento dos preços do farelo de soja, no município de Ilha Solteira acompanhou, em parte, a sazonalidade detectada para os preços médios pagos pelos produtores paulistas.

Palavras-chave: Sazonalidade. Preços do farelo de soja. Preços de fatores. Agronegócio.

\section{SEASONAL BEHAVIOUR PRICES OFSOYBEAN MEAL IN THE STATE OF SÃO PAULO AND PRICES IN ILHA SOLTEIRA (SP)}

\begin{abstract}
The issue of seasonality is an aspect that is of great importance, directly affecting the amount of products offered in the market and hence their prices. Therefore, farmers have to analyze the prices of factors of product into reduce the costs. Particularly for grain production, fertilizer prices are relevant. This study aimed to: i) analyze the behavior of prices of soybean meal in Ilha Solteira (SP); ii) compare them to averages of the State of São Paulo; and iii) calculate the seasonal index for the prices the State of São Paulo, using the Centralized Moving Geometric Mean Method, described by Hoffman (1980). It is concluded that: i) soybean prices in Ilha Solteira (SP) were always higher (except in July and August 2012) than soybean prices paid by producers of the State of São Paulo; ii) the average prices paid by producers in the State of São Paulo presented seasonal behavior, iii) the behavior of prices in Ilha Solteira has partially followed the sazonality detected by average prices paid for producers of the State of Sao Paulo.
\end{abstract}

Cultura Agronômica, Ilha Solteira, v.24, n.2, p.197-204, 2015 
Key words: Seasonality. Soybean meal prices. Factor prices. Agribusiness.

\section{INTRODUÇÃO}

À medida que há aumento na renda da população de um país ou região, há tendência de aumento no consumo de proteína de origem animal. Foi o que se verificou no Brasil, na metade dos anos de 1990, quando o frango tornou-se um dos símbolos do Plano Real. A classe média brasileira cresceu com a estabilização da moeda, e passou a representar $60 \%$ do crescimento do varejo. Assim, de acordo com Leitão (2011), o Brasil virou um mercado de consumo de massa. Em alguns momentos, um produto virou símbolo, como iogurte, frango. A mudança dos hábitos de consumo da população brasileira, como citado pela autora mencionada, acrescentou o frango como um alimento diário.

O aumento da procura por produtos de origem animal pode ocasionar uma pressão no mercado de determinados grãos. Particularmente quanto à soja, do lado da oferta desses produtos, Brasil e Estados Unidos representam, aproximadamente, 80\% da produção mundial da oleaginosa. Os preços do grão de soja são, então, afetados diretamente pela produção anual destes países. No que diz respeito ao farelo de soja, os preços mundiais apresentam comportamento que segue a produção da matéria prima; assim sua produção é acentuada por ocasião das colheitas do Hemisfério Sul (BARBOSA et al., 2000). Nos meses da entressafra da soja, a oferta do grão é baixa e os preços do farelo de soja apresentam-se relativamente superiores à média anual. Já na época da safra (fevereiro a junho) os preços diminuem, em vista da maior oferta do produto no mercado.

Em Ilha Solteira, no Noroeste do Estado de São Paulo, é possível que os valores de fretes influenciem os preços no município. Fator contribuinte para preços altos seria a concentração do mercado local — tanto pelo número de lojas de revenda (eram apenas quatro) quanto pela limitação na possibilidade de escolha de insumos (até indisponíveis às vezes).

Objetivou-se: i) analisar o comportamento de preços do farelo de soja no município de Ilha Solteira (SP), ii) compará-los a médias do Estado de São Paulo; e iii) calcular o índice de sazonal para o estado, pelo Método da Média Geométrica Móvel Centralizada.

\section{MATERIAL E MÉTODOS}

$\mathrm{Na}$ metodologia adotada, os preços do farelo de soja foram obtidos mensalmente, através de pesquisas nas agropecuárias do município de Ilha Solteira (SP). Foram tabulados utilizando-se o software Microsoft Excell. Em seguida, foram calculados os preços médios mensais do farelo de soja no município.

A partir da página do Instituto de Economia Agrícola (IEA, 2013) na internet, foram obtidos os preços médios mensais pagos pelos produtores do Estado de São Paulo, entre os anos de 2008 a 2012, e calculado o índice de sazonalidade de preços do farelo de soja para o estado. A metodologia adotada, no presente estudo, para análise da sazonalidade de preços 
para o Estado de São Paulo, é a proposta por Hoffman (1980), com a utilização da média geométrica móvel centralizada de 12 meses. Tal autor (1980) parte da consideração de que o preço é igual ao produto de três componentes:

$\mathrm{i}-$ uma tendência exponencial $\mathrm{AB}^{\mathrm{t}}=\exp \left\{\mathrm{a}+\mathrm{b}_{\mathrm{t}}\right)$;

ii - um componente adimensional $\varepsilon_{\mathrm{j}}$;

iii - um fator aleatório adimensional $U_{t}$.

Empregou a equação 1:

$$
P_{t}=P_{i j}=A B^{t} \varepsilon_{j} U_{t}
$$

Onde:

$\mathrm{P}=$ preço do produto;

$\mathrm{i}=$ indica o ano;

$\mathrm{j}=$ indica o mês.

O método descrito por Hoffman (1980) exige que sejam realizados os seguintes cálculos: (a) média geométrica móvel centralizada $\left(\mathrm{G}_{\mathrm{t}}\right)$, onde são eliminadas as variações sazonais e grande parte das variações aleatórias; (b) índices estacionais de preços, e (c) médias geométricas dos índices estacionais. De acordo com tal autor, os índices obtidos a partir dos preços correntes são muito semelhantes aos obtidos utilizando preços reais, não sendo necessário deflacionar os preços.

A análise a partir de médias móveis centradas (em um período n) consiste em suavizar as variações das séries por um processo de sucessivas médias. Quanto maior o número de termos utilizados para a média móvel, mais suavizada será a série resultante. Por suas características, a média móvel elimina as variações aleatórias e os movimentos sistemáticos que apresentam duração de tamanho n (HOFFMAN, 1980).

No caso de uma média móvel de um número par de termos $(\mathrm{k}=2 \lambda)$, como é o caso dos meses do ano, "um valor qualquer da média móvel não corresponde exatamente a nenhum dos termos da série dada. Por exemplo, uma média móvel de 4 termos corresponde a um intervalo entre o segundo e o terceiro termo considerado no seu cálculo. Para resolver esse problema, define-se a média aritmética móvel centralizada de $\mathrm{k}=2 \lambda$ termos" (HOFFMAN, 1980, p.335) multiplicando-se o primeiro e o último valor por 0,5. Com a aplicação de logaritmos neperianos em (1), a média dos preços a ser obtida será geométrica, sendo a média geométrica móvel centralizada de 12 meses para uma série de preços calculada pela equação 2 :

$$
G_{t}=\sqrt[12]{P_{t-6}^{0,5} \cdot P_{t-5} \ldots P_{t} \ldots P_{t+5} \cdot P_{t+6}^{0,5}}
$$

Onde:

$\mathrm{G}_{\mathrm{t}}=$ média geométrica móvel no mês $\mathrm{t}$;

$\mathrm{P}_{\mathrm{t}}=$ preço no mês $\mathrm{t}$;

$\mathrm{t}=$ mês em que a média é centralizada

Cultura Agronômica, Ilha Solteira, v.24, n.2, p.197-204, 2015 
Os índices estacionais dos valores pesquisados $\left(I_{t}\right)$ podem ser estimados dividindo-se o preço $\left(P_{t}\right)$ pela respectiva média geométrica $\left(G_{t}\right)$, e em seguida multiplicar por 100 , como apresentado na equação 3:

$$
I_{t}=\frac{P_{t}}{G_{t}} .100
$$

Em seguida foi determinada a média geométrica dos índices estacionais para cada mês, dada por $100 D_{j}^{*}$, onde o valor de $D_{j}^{*}$ é obtido através da equação 4 :

$$
D_{j}^{*}=\left(\prod_{i=1}^{n-1} D i j\right)^{\frac{1}{n-1}}
$$

Os valores de $\varepsilon_{\mathrm{j}}$ são denominados índices sazonais; eles caracterizam o padrão de variação estacional do preço do produto, são calculados pela equação 5:

$$
\varepsilon_{j}=\frac{D_{j}^{*}}{C}
$$

Onde:

$D_{j}^{*}=$ média geométrica dos índices estacionais de cada mês

$$
\begin{aligned}
& \mathrm{C}=\text { correção, calculada pela equação } 6: \\
& C=\left(\prod_{j=1}^{12} D_{j}^{*}\right)^{\frac{1}{12}}
\end{aligned}
$$

\section{RESULTADOS E DISCUSSÃO}

Na Tabela 1, apresentam-se os preços do farelo de soja, levantados nas agropecuárias de Ilha Solteira, os preços pagos pelos produtores no Estado de São Paulo e a diferença entre eles, para os anos de 2011, 2012 e 2013. A partir dos dados apresentados, é possível verificar que os comportamentos dos preços seguem a tendência de serem maiores no segundo semestre do ano, para os seis meses restantes de 2013, é provável que os preços apresentem-se maiores do que os meses anteriores.

Nota-se que há diferenças de preços mensais entre o município e o estado, em relação aos preços do farelo de soja (Tabela 1). É possível verificar que, na maior parte do ano, os preços do estado foram inferiores ao do município. Isso se dá, talvez, por se tratar de uma média de várias regiões paulistas; assim, determinadas regiões do estado podem apresentar preços mais baixos pela maior oferta local do produto. Outro fator que pode contribuir é à distância da região do Noroeste Paulista dos grandes centros de distribuição, influenciando no aumento dos preços devido o frete elevado. 
Tabela 1. Preços do farelo de soja (50 kg), em moeda corrente (Reais), levantados em Ilha Solteira versus preços no Estado de São Paulo e diferença entre eles, para abril de 2011 a junho de 2013

\begin{tabular}{cccccccccc}
\hline \multirow{2}{*}{ Mês } & \multicolumn{3}{c}{ Ilha Solteira } & \multicolumn{3}{c}{ Estado São Paulo } & \multicolumn{3}{c}{ Diferença } \\
\cline { 2 - 9 } & $\mathbf{2 0 1 1}$ & $\mathbf{2 0 1 2}$ & $\mathbf{2 0 1 3}$ & $\mathbf{2 0 1 1}$ & $\mathbf{2 0 1 2}$ & $\mathbf{2 0 1 3}$ & $\mathbf{2 0 1 1}$ & $\mathbf{2 0 1 2}$ & $\mathbf{2 0 1 3}$ \\
\hline Jan. & & 58,23 & 76,00 & & 36,50 & 61,50 & & 21,73 & 14,50 \\
Fev. & 53,63 & 75,00 & & 37,50 & 54,00 & & 16,13 & 21,00 \\
Mar. & & 53,63 & 76,00 & & 38,50 & 48,50 & & 15,13 & 27,50 \\
Abr. & 63,95 & 57,45 & 76,00 & 35,50 & 45,00 & 48,00 & 28,45 & 12,45 & 28,00 \\
Mai. & 62,00 & 55,45 & 76,00 & 33,50 & 47,50 & 50,50 & 28,50 & 7,95 & 25,50 \\
Jun. & 71,00 & 55,00 & 76,00 & 34,00 & 52,00 & 62,00 & 37,00 & 3,00 & 14,00 \\
Jul. & 62,63 & 55,00 & & 34,50 & 66,00 & & 28,13 & $-11,00$ & \\
Ago. & 62,63 & 45,00 & & 36,00 & 70,00 & & 26,63 & $-25,00$ & \\
Set. & 59,63 & 76,00 & & 36,50 & 74,00 & & 23,13 & 2,00 & \\
Out. & 59,63 & 76,00 & & 36,50 & 69,50 & & 23,13 & 6,50 & \\
Nov. & 59,48 & 76,00 & & 36,00 & 66,00 & & 23,48 & 10,00 & \\
Dez. & 59,48 & 76,00 & & 35,50 & 64,00 & & 23,98 & 12,00 & \\
\hline
\end{tabular}

Fonte: Dados da pesquisa e IEA (2013).

A menor diferença entre os preços, em termos absolutos, no ano de 2012, ocorreu em setembro. Destaca-se que, apenas nos meses de julho e agosto, os preços do município estiveram inferiores aos do estado. De janeiro a abril, os preços em Ilha Solteira foram bem superiores aos preços pagos pelos produtores paulistas (sendo acima de vinte reais no mês de janeiro). Entre os meses de junho e setembro, a diferença foi relativamente baixa, chegando a dois Reais em setembro. De setembro de 2012 a junho de 2013, o farelo de soja foi comercializado apenas por uma agropecuária de Ilha Solteira; os preços não sofreram alterações em tal período e mantiveram-se elevados. A alta é justificada, possivelmente, pela falta dos concorrentes durante esses meses na cidade.

A Figura 1 foi elaborada a partir do cálculo do índice sazonal do preço do farelo de soja (IEA, 2013) pagos pelos produtores de São Paulo. Utilizou-se a equação (5), proposta por Hoffman (1980). É notável a tendência de alta nos preços a partir de julho até fevereiro. $\mathrm{O}$ pico da alta dos índices ocorreu em janeiro e o menor índice em abril.

A partir da figura 1, é possível verificar que, entre os meses julho a fevereiro, o índice sazonal apresentou valores mais elevados (acima de 100) e ocorreu um pico em janeiro. Este período caracteriza-se pela entressafra da matéria-prima, o grão de soja, no Hemisfério Sul, onde, além do Brasil, tem também como importante produtor, a Argentina (terceiro produtor mundial da commodity), segundo dados da Food and Agriculture Organization - FAO (2013). Do lado da demanda por grãos, especificamente na produção de carnes bovina - em sistema de produção confinado ou semiconfinado - a utilização do farelo na dieta pode colaborar com a alta de preços no período de escassez de pastos. Os índices inferiores a 100 ocorreram de março a junho (com menor índice no mês de abril), refletindo a queda no nível dos preços do farelo de soja, possivelmente pela maior oferta da matéria prima.

Cultura Agronômica, Ilha Solteira, v.24, n.2, p.197-204, 2015 


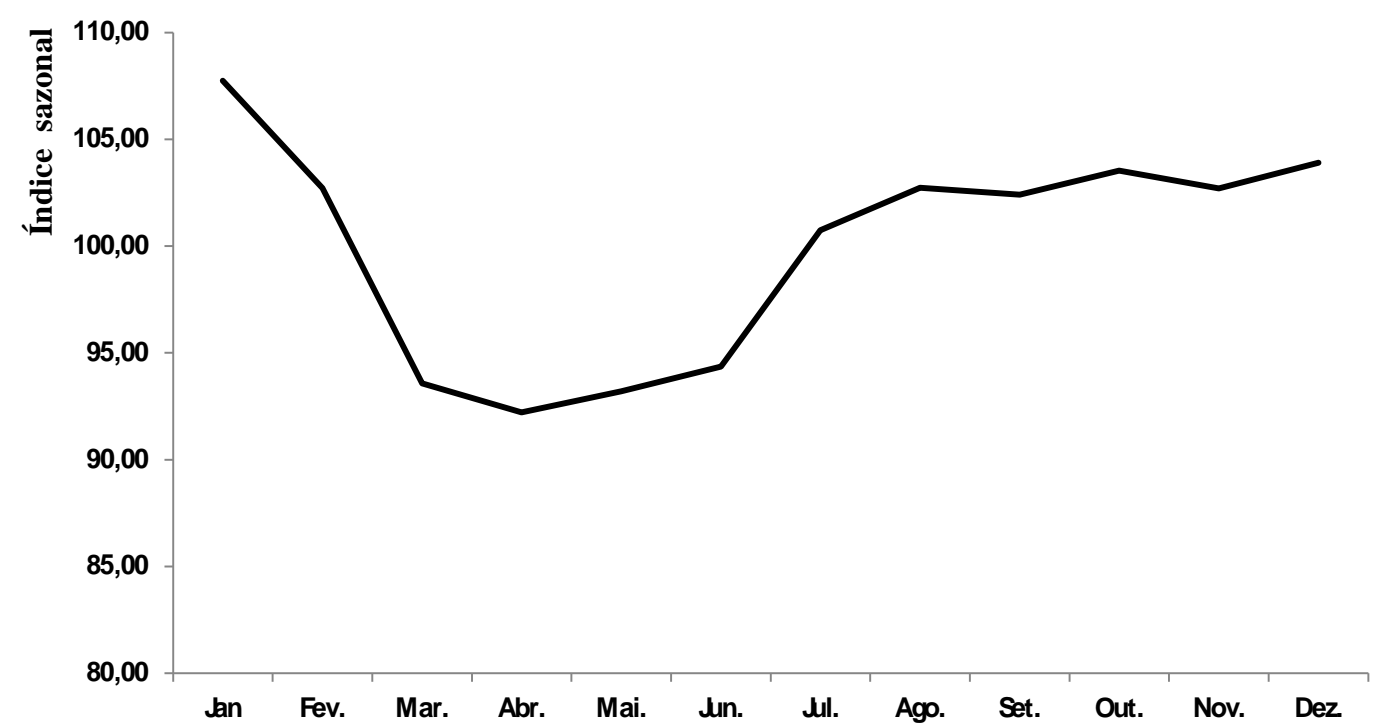

Figura 1. Índice sazonal do preço do preço do farelo de soja pagos pelos produtores de São Paulo, de 2008 a 2012

Corroborando com os resultados obtidos, Barbosa et al. (2000) ao estudarem o comportamento dos preços de soja e seus derivados nas exportações brasileiras, verificaram que em relação aos índices sazonais dos preços do farelo de soja brasileiro, esses superaram a média (100) entre julho e janeiro (com pico em dezembro), período de entressafra da matériaprima no Hemisfério Sul. Os índices inferiores à média ocorreram de fevereiro a junho (com vale em março) refletindo a queda no nível dos preços do farelo de soja pela maior oferta da matériaprima tanto no Brasil quanto na Argentina.

O cálculo do índice sazonal de preços do farelo de soja em Ilha Solteira não foi calculado, devido a não disponibilidade mínima de dados (apenas três anos, quando a literatura costuma utilizar pelo menos cinco). Assim, a comparação neste aspecto não é possível, mas pode-se afirmar que o comportamento dos preços do farelo de soja, no município de Ilha Solteira, acompanhou, em parte, a sazonalidade detectada para os preços médios pagos pelos produtores paulistas.

No presente trabalho foi realizado o deflacionamento prévio dos preços, porém os picos dos preços para Ilha Solteira se mantiveram os mesmos e os índices obtidos a partir dos preços correntes foram muito semelhantes aos obtidos utilizando preços reais. Este fato pode ser atribuído ao efeito da inflação ser captado pela média móvel, sendo praticamente eliminada quando, para se obter os índices estacionais, se divide o preço mensal pela correspondente média geométrica móvel centralizada (HOFFMAN, 1980). Cézar et al. (1995), ao estudar a variação estacional de séries de preço, sob diversas condições de inflação, verificaram que no período de 1969 a 1972, quando a inflação não foi muito irregular e tampouco muito alta, os índices sazonais médios dos preços foram quase que de mesma magnitude tanto para a série real quanto para a nominal. Concluindo que em períodos em que a inflação é baixa e não irregular, não se justifica realizar o deflacionamento dos preços para calcular a sazonalidade, assim como ocorre no período analisado (2008 a 2012).

Cultura Agronômica, Ilha Solteira, v.24, n.2, p.197-204, 2015 


\section{CONCLUSÕES}

A presente pesquisa concluiu que:

(1) os preços do farelo de soja, levantados nas lojas de produtos agropecuários do município de Ilha Solteira, no período de abril de 2011 a junho de 2013, foram superiores aos preços do farelo de soja pagos pelos produtores do Estado de São Paulo (exceto em julho e agosto de 2012) no mesmo período;

(2) os preços médios pagos pelo farelo de soja por produtores paulistas confirmaram o comportamento sazonal detectado por outros autores e, no presente estudo, identificado por maiores índices sazonais nos meses de janeiro e dezembro,

(3) o comportamento dos preços do farelo de soja, no município de Ilha Solteira no ano de 2012 acompanhou, em parte, a sazonalidade detectada para os preços médios pagos pelos produtores paulistas, excetuando-se os meses de janeiro que foi superior, e agosto que se apresentou relativamente inferior.

\section{AGRADECIMENTOS}

Os autores agradecem a PROEX/UNESP pelos recursos e bolsas oferecidos ao projeto de extensão "Levantamento de Preços de Insumos Agropecuários em Ilha Solteira", nos anos de 2011 a 2013.

\section{REFERÊNCIAS BIBLIOGRÁFICAS}

BARBOSA, M. Z.; FERREIRA, C. R. R. P. T.; FREITAS, S. M. Comportamento dos preços de soja e derivados nas exportações brasileiras no período de 1989-97. Informações Econômicas, São Paulo, v. 30, n. 2, p.25-30, 2000.

CÉZAR, S. A. G.; SUEYOSHI, M. L. S.; PINO, F. A.; FRANCISCO, V. L. F. S.; AMARAL, A. M. P. Ajustamento sazonal de preços com a inflação. Agricultura em São Paulo, São Paulo, v. 42, n. 2, p.39-63, 1995.

FOOD AND AGRICULTURE ORGANIZATION - FAO. Produção de produtos alimentares a agrícola. Disponível em: <http://faostat.fao.org/DesktopDefault.aspx?PageID=339\&lang=es>. Acesso em: 10 set. 2013.

HOFFMAN, R. Estatística para Economistas. São Paulo: Pioneira, 1980. 379 p.

INSTITUTO DE ECONOMIA AGRÍCOLA. Preços Médios Mensais Pagos pela Agricultura. Disponível em: <http://ciagri.iea.sp.gov.br/nia1/Precos_Medios.aspx?cod_sis=5>. Acesso em: 20 nov. 2013.

Cultura Agronômica, Ilha Solteira, v.24, n.2, p.197-204, 2015 
LEITÃO, M. Saga brasileira: a longa luta de um povo por sua moeda. 3 ed. Rio de Janeiro: Record, 2011. 475 p. 